\title{
Synthetic spectra of young starbursts: exploring the metallicity dependence
}

\author{
A. de Koter ${ }^{1}$, S.R. Heap ${ }^{2}$, D.J. Hillier ${ }^{3}$ and I. Hubeny ${ }^{4}$ \\ ${ }^{1}$ Astronomical Institute Anton Pannekoek, University of Amsterdam, \\ Kruislaan 403, NL-1098 SJ, Amsterdam, the Netherlands \\ ${ }^{2}$ Laboratory for Astronomy and Solar Physics, \\ Code 681, Goddard Space Flight Center, Greenbelt, MD 20771, USA \\ ${ }^{3}$ Department of Physics and Astronomy, University of Pittsburgh, \\ $P A$ 15260, USA \\ ${ }^{4}$ AURA, National Optical Astronomy Observatories, \\ Code 681, Goddard Space Flight Center, Greenbelt, MD 20771, USA
}

\begin{abstract}
We present first results of fully synthetic UV spectra of young starbursts. With this we mean that the spectra used to model the OB and WN stars result from unified non-LTE model atmospheres, in which stellar winds are accounted for. An important aim of this project is to construct meaningful models for young starbursts at low metallicities. We discuss the presence of He II emission as a function of maximum initial mass and age of the burst.
\end{abstract}

\section{Synthetic starburst models}

Massive stars are an important diagnostic in understanding the rate and mechanism of star formation in galaxies. Starbursts containing massive stars occur in a wide variety of environments, from extremely metal-poor systems, such as I Zw 18, to super-metal-rich systems, e.g., M 83. Radiation driven wind theory predicts that metallicity will influence the rate with which massive stars loose mass: higher $Z$ yields higher $\dot{M}$. With increasing metallicity comes a different course of evolution and (possibly) ultimate fate of the star, and a different return, chemical yield, and radiative \& dynamical input to the surrounding medium.

The problem of constructing a meaningful model of evolution synthesis of massive stars in starbursts is extremely complex as it brings together complicated fields such as star formation, stellar evolution and stellar atmospheres. Our method is unique in that it employs synthetic spectra for hot stars with mass loss. This allows one to predict how metallicity influences the observed spectrum of a starburst. We present first results of these predictions.

The energy distributions presented in the figure are computed using the unified atmosphere code ISA-WIND of de Koter et al. (1998). Wind properties may be specified. We adopt mass loss rates consistent with those used in the Meynet et al. (1994) evolutionairy tracks and a metallicity scaling $\dot{M} \propto Z^{0.5}$. Chemical species included are $\mathrm{H}, \mathrm{He}, \mathrm{C}, \mathrm{N}, \mathrm{O}, \mathrm{Si}$ and $\mathrm{S}$. The spectra account for the blending by iron-group elements using a generalized form of the Modified Nebular Approximation (Schmutz 1991). 

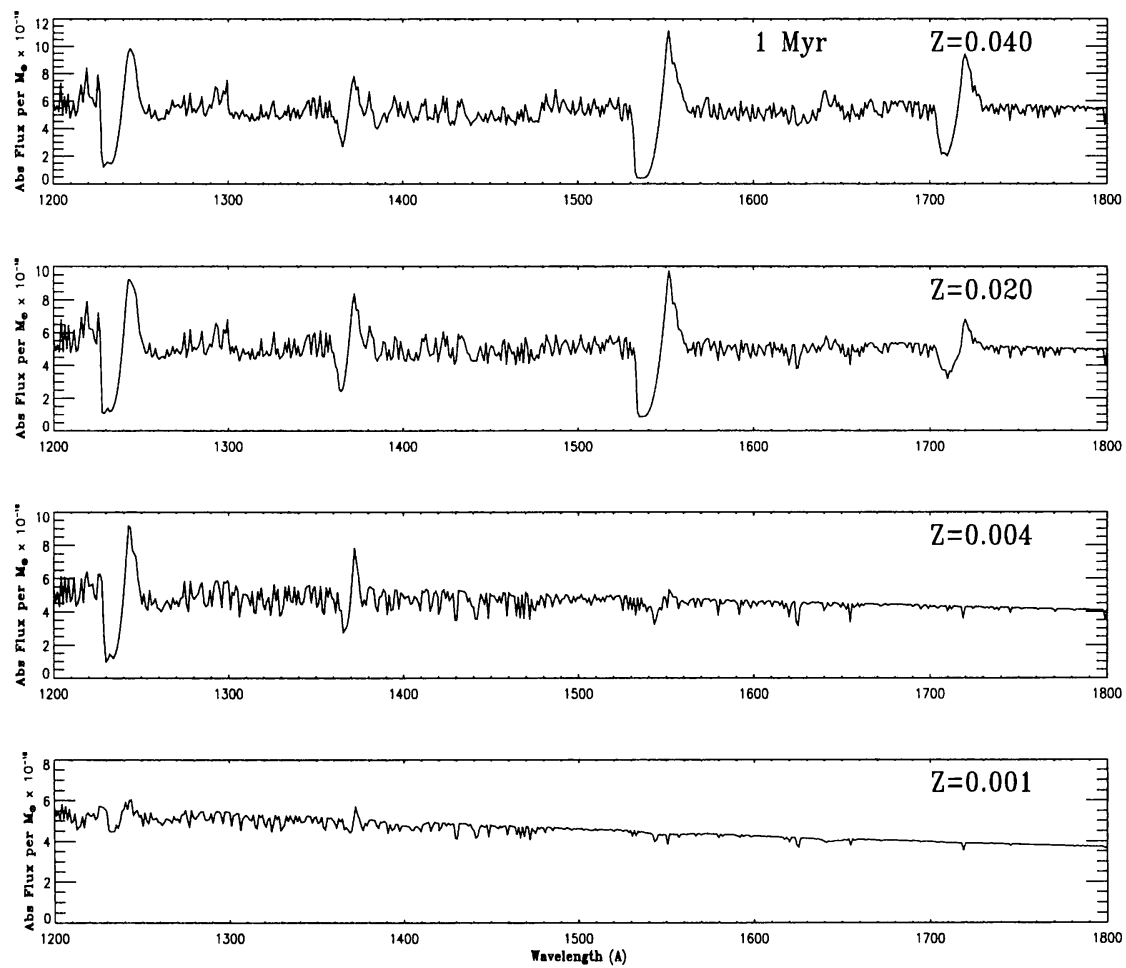

Figure 1. Computed UV spectra of a star cluster at $1 \mathrm{Myr}$ for the case of instantaneous star formation, Salpeter IMF, and $M_{\max }=120 \mathrm{M}_{\odot}$. Four different metallicities are shown.

The figure shows the influence of metallicity on spectral appearance. The presence of He II $\lambda 1640$ in emission is clearly seen for the highest $Z$ and possibly for the case $Z=0.020$. This emission is correlated with that of optical He II $\lambda 4686$ emission defining Wolf-Rayet clusters or galaxies. It is a strong function of the maximum initial stellar mass in the burst, $M_{\max }$, and of age. At the onset of the burst, no He II emission is seen because of the rather steep decrease in $\dot{M}$ towards higher $T_{\text {eff. }}$ At $1 \mathrm{Myr}$, emission is seen at $M_{\max } \geq 85 \mathrm{M}_{\odot}$ for $Z=0.040$ and at $M_{\max } \geq 120 \mathrm{M}_{\odot}$ for $Z=0.020$. At $2 \mathrm{Myr}$, emission is present at $M_{\max } \geq 60,85,120$, and $120 \mathrm{M}_{\odot}$ for $Z=0.040,0.020,0.004$ and 0.001 , respectively. At $Z=0.040$ and 0.020 , the initially $120 \mathrm{M}_{\odot}$ stars have evolved into WNL stars. In all other cases, the He II emission is due to Of stars.

\section{References}

de Koter, A., Heap, S.R., Hubeny, I. 1998, ApJ 509, 879

Meynet, G., Maeder, A., Schaller, G., Schaerer, D., Charbonnel, C. 1994,A\&AS 103, 97

Schmutz, W. 1991, in: L. Crivellari, I. Hubeny \& D.G. Hummer (eds.), Stellar Atmospheres: Beyond Classical Models, NATO ASI Series C-341, 191 